\title{
Simultaneous Infrared Spectroscopy, Raman Spectroscopy and Luminescence Sensing: A Multi-Spectroscopic Analytical Platform
}

\author{
Sarah Klingler ${ }^{\mathrm{a}}$, Julian Hniopek ${ }^{\mathrm{b}, \mathrm{c}}$, Robert Stach ${ }^{\mathrm{d}}$, Michael Schmittc ${ }^{\mathrm{c}}$, Jürgen Popp ${ }^{\mathrm{b}, \mathrm{c}}$ and Boris \\ Mizaikoff ${ }^{a, d, *}$ \\ a Institute of Analytical and Bioanalytical Chemistry, Ulm University, Albert-Einstein-Allee 11, $89081 \mathrm{Ulm}$, Germany \\ ${ }^{b}$ Department of Spectroscopy/Imaging, Leibniz-Institute of Photonic Technologies, Jena, Germany \\ c Institute of Physical Chemistry \& Abbe Center of Photonics, Friedrich Schiller University Jena, Jena, Germany \\ ${ }^{d}$ Hahn-Schickard, Sedanstraße 14, 89077 Ulm, Germany
}

KEYWORDS infrared spectroscopy, attenuated total reflection, ATR, Raman spectroscopy, luminescence sensing, luminescence quenching, multi-spectroscopic platform, combined spectroscopic methods, $\mathrm{H}_{2} \mathrm{O}, \mathrm{D}_{2} \mathrm{O}$, ammonium sulfate, oxygen

\begin{abstract}
Scientific questions in fields such as catalysis, monitoring of biological processes or environmental chemistry demand for analytical technologies combining orthogonal spectroscopies. Combined spectroscopic concepts facilitate in-situ on-line monitoring of dynamic processes providing for a better understanding of the involved reaction pathways. In the present study, a low-liquid-volume multi-spectroscopic platform was developed based on infrared attenuated total reflection (IR-ATR) spectroscopy combined with Raman spectroscopy and luminescence sensing. For demonstrating the measurement capabilities, exemplary analyte systems including water / heavy water and aqueous solutions of ammonium sulfate were analyzed as proof-of-principle studies. It was successfully demonstrated that three optical techniques may be integrated into a single analytical platform without interference providing synchronized and complementary datasets by probing the same minute sample volume. In addition, the developed assembly provides a gas-tight lid sealing the headspace above the probed liquid for monitoring the concentration of molecular oxygen also in the gas phase via luminescence quenching. Hence, the entire assembly may be operated at inert conditions, as required for example during the analysis of photocatalytic processes.
\end{abstract}

\section{INTRODUCTION}

For many state-of-the-art analytical questions including reaction pathway monitoring during photocatalysis or biological processes, rapid and time-resolved tracking of molecular structures, composition and quantities is a prerequisite. Mid-infrared (MIR) spectroscopy has matured into one of the most prevalent analytical techniques for monitoring molecular processes owing to its inherent selectivity, non-destructiveness, and rapid data acquisition capabilities. Thus, molecular information on a wide range of organic and inorganic species may be obtained. Consequently, IR-ATR is frequently used as an analytical tool for the detection, identification, and quantification of molecules in the gas, liquid, and solid phase. ${ }^{1,2}$ Due to the excitation of specific vibrational, ro-vibrational, and rotational modes, characteristic spectral patterns are obtained enabling qualitative and quantitative analysis, and providing access to chemical and structural characteristics, i.e., 'fingerprinting' any molecular species. Using Fourier transform infrared (FTIR) spectrometers facilitates timeresolved studies for in-situ and on-line monitoring, e.g., in process control, during reaction pathway elucidation, and in environmental analysis. ${ }^{1,3-5}$

Attenuated total reflection spectroscopy is among the most flexible sampling techniques in this domain, esecially for condensed phase applications with pronounced background matrix absorptions (e.g., water in aqueous solutions), as the evanescent field only probes few micrometers into the sample above the internal reflection element (IRE) surface. ${ }^{6-8}$ While a variety of IRE materials are available, diamond is most beneficial taking advantage of its chemical inertness (e.g., against acids and bases), physical resilience, and mechanical robustness next to its advantageous refractive index providing a useful penetration depth of the evanescent field. ${ }^{7,9}$

During the present study, we have developed an analytical assembly combining the benefits of IR-ATR spectroscopy with its complementary vibrational spectroscopic counterpart, i.e., Raman spectroscopy. Next to IR spectroscopy, Raman spectroscopy has matured into a likewise commonly applied vibrational spectroscopic technique in chemical and biological analysis. ${ }^{7,10,11}$ As both 
techniques are based on different physical principles concerning the excitation of molecular vibrations (i.e., IR spectroscopy relies on a change of the molecular dipole moment, while Raman scattering requires a change in molecular polarizability), vibrations may be active or inactive in IR vs. Raman or - alternatively - less or more pronounced in either technique. Thus, IR and Raman spectroscopy provide complementary molecular characteristics. ${ }^{10-13}$

Besides Raman and IR-ATR spectroscopy, a third optical technique was integrated into the multi-spectroscopic platform, i.e., luminescence sensing. This adds the opportunity of precisely quantifying the oxygen concentrations in the gas and the liquid phase, which plays an important role in a wide variety of monitoring scenarios including industrial process monitoring, environmental monitoring (e.g., quality control of water ${ }^{14,15}$ ), and in photocatalysis (e.g., determination of the turn-over-number (TON) during water oxidation reactions $\left.{ }^{16}\right) .{ }^{17-19}$ As oxygen does not provide IR-active vibrations, and Raman spectroscopy is frequently not sensitive enough for gas phase oxygen sensing - and even less so for analyzing dissolved molecular oxygen - an additional optical technique was integrated, i.e., oxygen sensing based on luminescence quenching effects. Dynamic quenching of luminescence of appropriate dyes has matured into one of the most commonly applied techniques for the detection and quantification of molecular oxygen, in particular using fiberoptically coupled sensing techniques with tip-immobilized dyes. Next to an exceptionally fast response time, this detection principle does not consume oxygen, is inherently selective and robust, and enables measurements even in hard-toreach places. Hence, luminescence-based oxygen sensors have several advantages vs. conventional techniques. ${ }^{12,17-22}$

The particular development goal of the present study was establishing a methodology that facilitates improving fundamental understanding on photocatalytic reaction pathways along with the involved molecular changes of the photocatalyst and/or the embedding immobilization matrix. As each of the presented in-situ spectroscopic techniques (i.e., IR, Raman, and luminescence quenching) provide individual advantages and disadvantages, the combination offers a powerful tool to overcome the drawbacks, and to benefit from the strengths of each method. ${ }^{23}$ Raman spectroscopy provides advantages such as the comparative insensitivity towards water background signals. ${ }^{24-28}$ This complements IR-ATR spectroscopy that is much more affected by aqueous matrices. ${ }^{11}$ However, depending on the excitation wavelength Raman spectroscopy may suffer from a significant fluorescence background convoluting the vibrational signatures. ${ }^{10}$ Consequently, combining IR and Raman spectroscopy provides orthogonal information even facilitating potential insitu validation when simultaneously operated the same sample. ${ }^{24}$

For clarification of reaction mechanisms in, e.g., photocatalytic reactions and for determination of active species in-situ spectroscopic methods and especially their combination are of relevance for future investigations at in-operando conditions. In-situ spectroscopy enables deriving information on reaction intermediates and structural changes during the reaction with temporal resolution. ${ }^{23,29,30}$ Useful applications of combined in-situ spectroscopic techniques (i.e., involving Raman, UV-Vis, FBRM, IR-ATR, etc.) have been shown for a variety of scenarios providing supplementary information on reaction pathways and in process control (e.g., crystallization processes $^{25,28}$, catalytic reactions ${ }^{24,23}$, polymorphic transformations ${ }^{26,31-33}$, etc.). 'Gold standard' methods such as mass spectrometry, X-ray absorption spectroscopic methods (i.e., X-ray absorption near edge structure (XANES) or extended X-ray absorption fine structure (EXAFS)) or magnetic resonance spectroscopic methods (i.e., electron para-magnetic resonance (EPR), and NMR) were applied for studying several types of catalytic and (bio-)chemical reactions in the liquid phase, yet are in part limited by extended measurement times and lack in compactness and robustness for integration. ${ }^{23,24,34}$

Especially 2D data correlation methods benefit from the combination of orthogonal techniques. As a relatively young research field introduced by Noda in 1993, homoand heterospectral 2D correlation techniques have evolved into effective and powerful tools for extracting spectroscopic information from complex data sets and minute molecular changes. By spreading the spectral information across two dimensions, systematic changes in data sets recorded under external perturbation may readily be obtained. ${ }^{35}$ Earlier research by our team and collaborators has successfully shown the clarification of catalytic reaction pathways via the heterogeneous correlation of Raman and IR-ATR spectra, however, not yet simultaneously recorded at the same sample. ${ }^{36}$ Hence, ensuring comparable reaction conditions (e.g., signal integration time, sample preparation, temperature, ambient conditions, etc.) results in extended experimental efforts. This further motivates the development of a combined platforms integrating different spectroscopic techniques for ensuring the same analysis conditions facilitating data fusion and/or correlation.

In the present approach, a multi-spectroscopic measurement platform was developed based on a singlebounce diamond IRE ATR assembly coupled to a conventional FTIR spectrometer integrating fiberoptic Raman spectroscopy into the sampled volume. In addition, fiberoptically coupled luminescence quenching sensors for determining oxygen was integrated for monitoring the integrity of the lid sealing, and the stability of headspace conditions. Background stability is essential, in particular if small changes - here, in oxygen concentration - should be observed, e.g., during future photocatalytic conversions. For demonstrating the utility of the developed assembly, two exemplary yet relevant measurement scenarios were selected. Mixtures of $\mathrm{H}_{2} \mathrm{O}$ and $\mathrm{D}_{2} \mathrm{O}$ were evaluated, as deuteration is an important modulator for the elucidation of reaction pathways. ${ }^{5,37}$ Additionally, various concentrations of ammonium sulphate were analyzed owing to its vibrational spectrum with differently pronounced vibrational features in IR and Raman spectra along with its relevance in environmental sensing scenarios (e.g., as component in fertilizers). ${ }^{38,39}$

\section{EXPERIMENTAL SECTION}


Multi-spectroscopic measurement platform. Figure 1a shows the developed multi-spectroscopic measurement platform. The system was fabricated from stainless steel (VA 1.4301) consisting out of two parts: (i) a lid (A) with five tapped holes (M6) (J) for inserting different fiberoptically coupled sensors gas-tight via thread adapters, and (ii) a lower part (B) with a circular aperture with conical shape $(4.5 \mathrm{~mm}$ diameter) at the bottom $(\mathrm{K})$ (detail see Figure 1b) facilitating IR-ATR measurement via a single-bounce diamond IRE. An additional port $(12 \mathrm{~mm}$ diameter) was included for housing the Raman probe. For the insertion of the sensors, the lid provides six milled surfaces (at an angle of $25^{\circ}$ each) providing a flat surface for sealing via O-rings and ensuring a suitable probe angle for the analysis. Stainless steel provides robustness against most chemicals (e.g., acids, etc.), oxidation stability and corrosion resistance, while also avoiding background fluorescence that may affect the Raman measurements. The fluidic cell requires a small sample volume of only $7 \mathrm{~mL}$. All parts were sealed gas-tight using Viton (FPM75) O-rings (black rings). For inserting the Raman probe (F) (RIP-RPB-785-FC-SMA, Ocean Optics B. V, EW Duiven, Netherlands), a customized adapter (E) was developed and fabricated from stainless steel (VA 1.4301), and sealed via an optical window made from sapphire $(G)$ (0.4 mm thickness $\times 7.5 \mathrm{~mm}$ diameter) glued with epoxy resin. As the Raman probe has a converging lens with a focal length of $7.5 \mathrm{~mm}$, the adapter had to be inserted into the liquid for optimal sensor response. For inserting other sensors in a gas-tight fashion, customized adapters were developed and fabricated $(\mathrm{C}, \mathrm{H})$ as needed. For inserting the temperature sensor (I), again stainless steel VA $1.4301(\mathrm{H})$ was used ensuring thermal conductivity and chemical stability. For integrating two fiberoptic oxygen sensors ( $D$; one for the gas phase, one for the liquid phase) PEEK (C) was utilized. The temperature sensor (Pt100 temperature probe, TDIP15, PyroScience $\mathrm{GmbH}$, Aachen, Germany) (I) was included as oxygen measurements via luminescence quenching are strongly dependent on temperature. ${ }^{19}$ The multi-spectroscopic measurement platform was established adapting a Platinum Diamond ATR module (Bruker Optik GmbH, Ettlingen, Germany) connected to a compact FTIR spectrometer (Alpha I OEM, Bruker Optik GmbH, Ettlingen, Germany) shown in Figure 1c.

b)

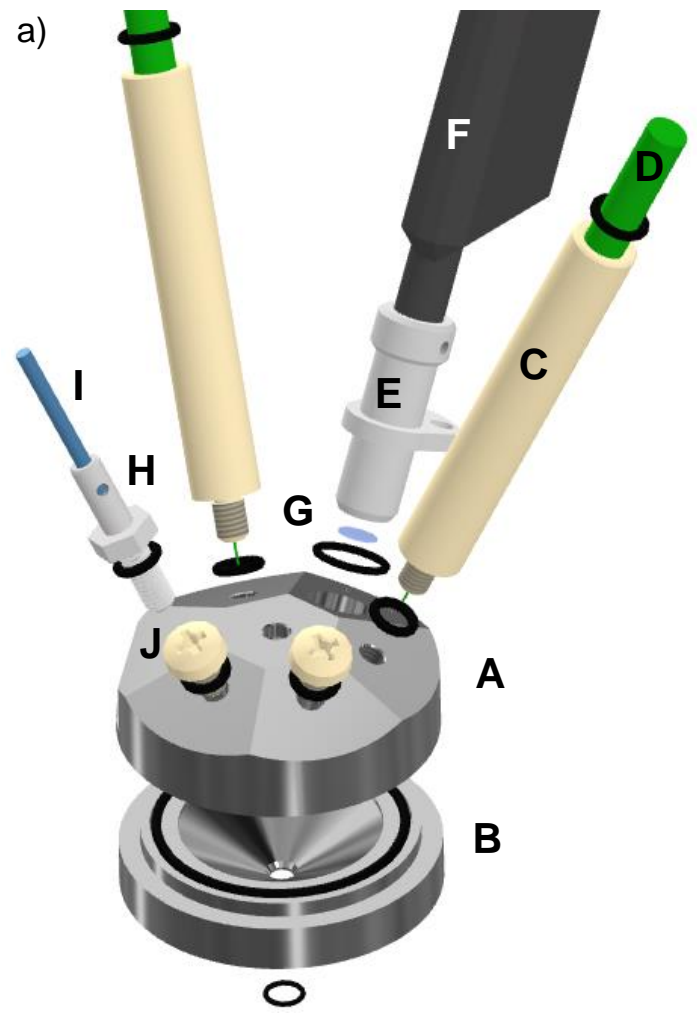

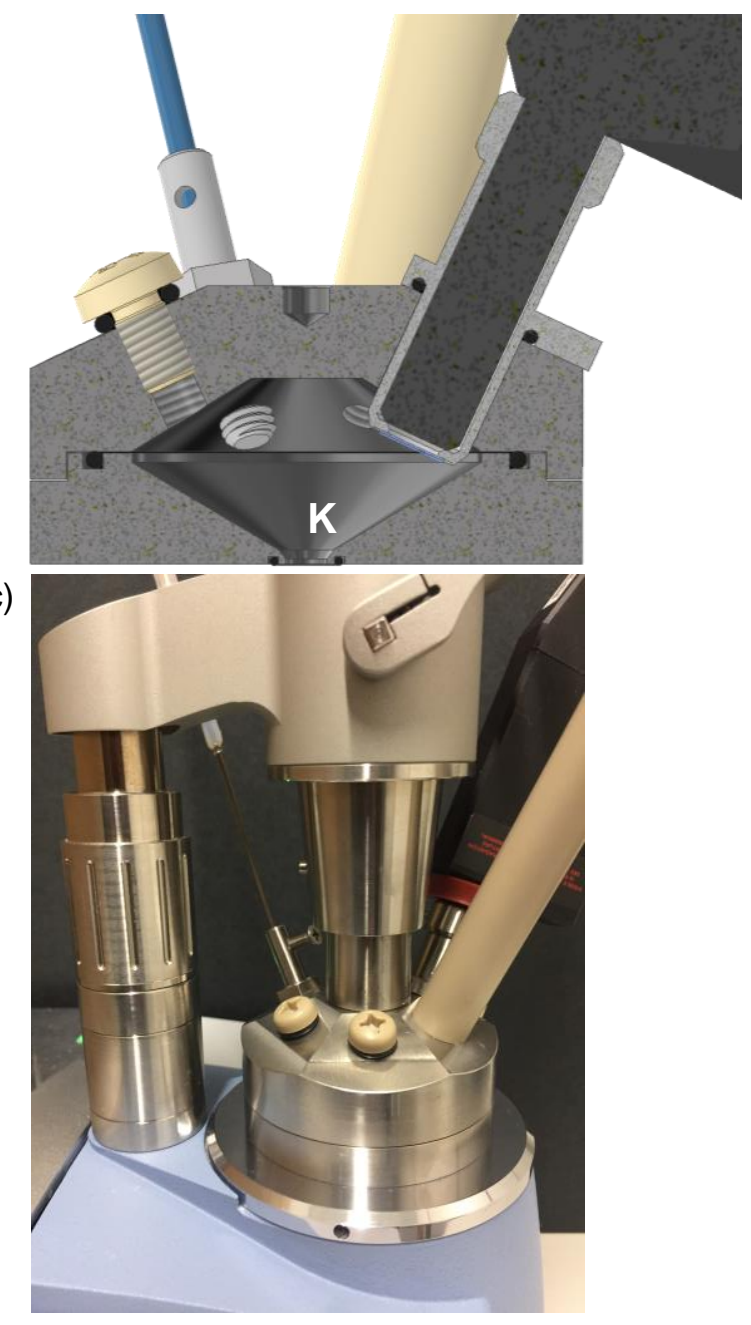

Figure 1. Schematic (CAD rendering) of the developed multi-spectroscopic measurement assembly. Exploded drawing (a), cross-sectional view of the sample chamber (b), and image of the assembly (c) attached to a compact FTIR spectrometer. 
Measurement procedure. An IR background spectrum was recorded after $7 \mathrm{~mL}$ sample solution was filled into the measurement cell via a pipette. The cell was then sealed with a Viton O-ring and a screw. A Raman dark background spectrum was also collected before turning on the laser and simultaneously executing all three optical measurements.

For the calibration of the oxygen sensors, a 2-point customized calibration procedure was executed. For the gas phase detection and for setting the $0 \% \mathrm{O}_{2}$ point, an $\mathrm{N}_{2}$ atmosphere $\left(99.999 \% \mathrm{~N}_{2}\right.$, class $5.0, \mathrm{MTI}$ IndustrieGase AG, Neu-Ulm, Germany) was used; for the second point, synthetic air $\left(20.5 \% \mathrm{O}_{2}, 79.5 \% \mathrm{~N}_{2}\right.$, MTI IndustrieGase AG, Neu-Ulm, Germany) was utilized. For the liquid phase oxygen calibration, $\mathrm{N}_{2}$ was used to sparge deionized water for approx. $6 \mathrm{~min}$. For setting the zero-calibration-point, the gas flow was turned off and a hold time was executed until no gas bubbles were evident. Afterwards, the aqueous matrix was purged with synthetic air and the same procedure was repeated to set the second calibration point.

Data acquisition. For IR data acquisition, the software package OPUS 8.5 (Bruker Optik GmbH, Ettlingen, Germany) was utilized. The IR spectra were recorded via a room temperature deuterated L-alanine doped triglycine sulfate (DLaTGS) detector in the spectral range of 400 to $4000 \mathrm{~cm}^{-1}$ at a spectral resolution of $2 \mathrm{~cm}^{-1}$, using a Blackman-Harris three-term apodization and a zero-filling factor of 2 averaging 52 scans for each spectrum. For Raman data acquisition and processing, a Maya2000Pro spectrometer (grating type 600, slit size: $25 \mu \mathrm{m}$ ) and the software OceanView 1.6.7 (both Ocean Optics B. V, EW Duiven, Netherlands) were used with an integration time of $4000 \mathrm{~ms}$ for each scan averaging 6 scans, which resulted in a total integration time of $24 \mathrm{~s}$. The excitation wavelength was $785 \mathrm{~nm}$ at a laser power of $900 \mathrm{~mW}$ (Ocean Optics B. V, EW Duiven, Netherlands). A boxcar smoothing width of 5 was used averaging over adjacent detector elements (i.e., averaging each data point with 5 points to the left and the right). The signals of the two luminescence sensors (Oxygen Retractable Microsensors OXR50, PyroScience GmbH, Aachen, Germany) were recorded using the software Pyro Oxygen Logger 3.31.8 (PyroScience GmbH, Aachen, Germany) in continuous data acquisition mode. Every $3 \mathrm{~s}$ one data point was recorded with data smoothing of 3 in both, gas and liquid phase processed by the FireSting-PRO (FSPRO-4) Oxygen Meter (PyroScience GmbH, Aachen, Germany).

$\mathrm{IR}$, Raman, and luminescence measurements were started simultaneously.

Data evaluation. Data evaluation was performed using the spectroscopy software package eFTIR (Operant LLC) and Origin 2021 (OriginLab). 2D correlation analysis was performed using GNU R and custom $R$ packages, which have been described in detail elsewhere..$^{40,41}$ As the reference spectrum, the mean spectrum of the data set was selected in all cases.

For evaluating different mixtures of $\mathrm{H}_{2} \mathrm{O} / \mathrm{D}_{2} \mathrm{O}$ $\left(100 \% \mathrm{H}_{2} \mathrm{O}, 100 \% \mathrm{D}_{2} \mathrm{O}, \mathrm{H}_{2} \mathrm{O}: \mathrm{D}_{2} \mathrm{O} 9: 1,3: 1,1: 1,1: 3\right)$ each measurement for the particular ratio was repeated five times.

For ammonium sulfate studies, each measurement was repeated ten times, and six different concentrations were analyzed $(0 \mathrm{M}, 0.05 \mathrm{M}, 0.10 \mathrm{M}, 0.25 \mathrm{M}, 0.50 \mathrm{M}, 0.75 \mathrm{M}$, $1.00 \mathrm{M})$.

For IR and Raman spectral data evaluation of sulfate $\left(\mathrm{SO}_{4}{ }^{2}\right)$ and ammonium $\left(\mathrm{NH}_{4}{ }^{+}\right)$, respectively, as well as for $\mathrm{H}_{2} \mathrm{O}$ and $\mathrm{D}_{2} \mathrm{O}$ concentrations an integration method was developed. The respective spectral regions are summarized in Table $1 .{ }^{42-46}$

Table 1. Parameters for IR and Raman spectral evaluation

\begin{tabular}{lccc} 
method & band & $\begin{array}{c}\text { integration / } \\
\mathrm{cm}^{-1}\end{array}$ & $\begin{array}{c}\text { baseline correction / } \\
\mathrm{cm}^{-1}\end{array}$ \\
\hline $\mathrm{IR}$ & $\mathrm{dSO}_{4}{ }^{2-}$ & $1148-1058$ & $1167-1023$ \\
& $\mathrm{NH}_{4}{ }^{+}$ & $1484-1424$ & $1523-1380$ \\
& $\mathrm{D}_{2} \mathrm{O}^{\mathrm{a}}$ & $2767-2181$ & $2808-2170$ \\
\multirow{5}{*}{ Raman } & $\mathrm{H}_{2} \mathrm{O}^{\mathrm{a}}$ & $3534-3058$ & $2752-3802$ \\
& ${ }^{\mathrm{S}} \mathrm{SO}_{4}{ }^{2-}$ & $999-956$ & $1032-901$ \\
& $\mathrm{NH}_{4}{ }^{+}$ & - & - \\
& $\mathrm{D}_{2} \mathrm{O}^{\mathrm{a}}$ & $2768-2242$ & $2807-2169$ \\
& $\mathrm{H}_{2} \mathrm{O}^{\mathrm{a}}$ & $3654-2930$ & $3771-2884$
\end{tabular}

For evaluating the performance of the developed measurement platform with regard to water and deuterium oxide mixtures, also the intensity ratios were determined by calculating the ratio of the mean value of the peak intensity for $100 \% \mathrm{H}_{2} \mathrm{O}$ and $\mathrm{D}_{2} \mathrm{O}$ for IR and Raman.

The mean averaged values of the integrated peak areas for $\mathrm{SO}_{4}{ }^{2-}$ and $\mathrm{NH}_{4}{ }^{+}$were then normalized with respect to the maximum sulfate integral for better comparison and plotted against the concentration for performing a linear regression.

The determination of the limit of detection (LOD) and limit of quantification (LOQ) for both systems was performed according to the IUPAC $3 \sigma / 10 \sigma$ criteria.

Chemicals. Ammonium sulfate $\left(\left(\mathrm{NH}_{4}\right)_{2} \mathrm{SO}_{4}\right.$, for biochemistry) was obtained from Merck (Darmstadt, Germany); deuterium oxide $\left(\mathrm{D}_{2} \mathrm{O}, 99.9 \%\right)$ in analytical quality was purchased from Deutero $\mathrm{GmbH}$ (Kastellaun, Germany). Both commercially available chemicals were used without further purification. For dilutions, deionized water was utilized. 


\section{RESULTS AND DISCUSSION}

The multi-spectroscopic measurement platform was evaluated in terms of functionality and during proof-of-principle studies providing orthogonal data, respectively.

Analysis of deuterium oxide and water. Deuteration is a common approach enabling non-radioactive isotope labeling. Thereby, complex reaction pathways or biological roots become traceable, especially also with vibrational spectroscopic techniques. In order to study the capability of the multi-spectroscopic platform for isotope tracking, different volume mixtures of $\mathrm{D}_{2} \mathrm{O}$ and $\mathrm{H}_{2} \mathrm{O}$ were analyzed. Figure $2 \mathrm{a}$ and $\mathrm{b}$ show the absorption and Raman spectrum of different ratios. The rather broad bands can be explained by the coordination of water molecules in solution, i.e., effects of hydrogen bonding and intermolecular coupling (i.e., environmental broadening). ${ }^{43,47}$ The occurrence of the double peak for the symmetric stretching vibrations in the resulting IR and Raman spectra - for $\mathrm{D}_{2} \mathrm{O}^{\mathrm{a}}$ at 2469 (IR) and $2484 \mathrm{~cm}^{-1}$ (Raman), respectively and $\mathrm{H}_{2} \mathrm{O}^{\mathrm{a}} 3323$ (IR) and $3235 \mathrm{~cm}^{-1}$ (Raman) - at high $\mathrm{D}_{2} \mathrm{O}$ or $\mathrm{H}_{2} \mathrm{O}$ concentrations, respectively, is a result of the existence of non-hydrogen bonded (NHB) and hydrogenbonded (HB) molecules. ${ }^{43}$ As evident in the IR spectra, the intensity ratio of $\mathrm{H}_{2} \mathrm{O}$ in comparison to $\mathrm{D}_{2} \mathrm{O}$ equals 0.997 comprising similar IR sensitivities (i.e., change in dipole moment). ${ }^{43,47}$ In contrast, the intensity ratio $\mathrm{H}_{2} \mathrm{O}$ to $\mathrm{D}_{2} \mathrm{O}$ resulting for the Raman studies is 0.157 indicating that the Raman sensitivity to $\mathrm{D}_{2} \mathrm{O}$ is more than six-times higher than for $\mathrm{H}_{2} \mathrm{O}$ owing to the decrease in intrinsic scattering efficiency at higher wavenumbers (i.e., $\omega^{4}$ dependency) and the decreased detector sensitivity for exceedingly long wavelengths $\left(3000 \mathrm{~cm}^{-1}=1025 \mathrm{~nm} @ 785 \mathrm{~nm}\right.$ excitation wavelengths). By evaluating the LOD and LOQ, this behavior is again evident, as summarized in Table 2. The LOD of $\mathrm{D}_{2} \mathrm{O}$ for Raman measurements is twice as high as for IR-ATR experiments, and almost 8-times higher than for $\mathrm{H}_{2} \mathrm{O}$. This indicates that the Raman measurements are less affected by high water content within the sample matrix. Furthermore, the Grotthuss mechanism (i.e., the molecular pathway through which protons or deuterons are exchanged between water molecules) could indeed be tracked via the HDO signal. ${ }^{37}$

Table 2. Comparison of the LOD and $L O Q$ for $\mathrm{H}_{2} \mathrm{O}$ and $\mathrm{D}_{2} \mathrm{O}$ for Raman and IR measurements $(n=5)$

\begin{tabular}{lccc} 
method & band & LOD / \% & LOQ / \% \\
\hline IR & $\mathrm{D}_{2} \mathrm{O}^{\mathrm{a}}$ & 1.5 & 4.4 \\
\multirow{4}{*}{ Raman } & $\mathrm{H}_{2} \mathrm{O}^{\mathrm{a}}$ & 2.1 & 6.4 \\
& $\mathrm{D}_{2} \mathrm{O}^{\mathrm{a}}$ & 0.7 & 2.2 \\
& $\mathrm{H}_{2} \mathrm{O}^{\mathrm{a}}$ & 5.5 & 16.7
\end{tabular}

Isotope labeling facilitates a better understanding of reaction pathways, which clearly benefits from a combined IR/Raman approach. The benefit of labeling via the exchange of deuterium and hydrogen atoms has been demonstrated in a variety of scenarios using IR-ATR spectroscopy, e.g., for elucidating reaction pathways in environmental, biochemical or catalytic reactions. ${ }^{5,37}$ Deuterium labeling appears even more effective for Raman measurements, as the $\mathrm{D}_{2} \mathrm{O}$ band is significantly more intense and pronounced in comparison to the $\mathrm{OH}$ stretching vibration in IR. In contrast, the $\mathrm{OH}$ stretching vibration is of the same intensity as the OD stretching vibration. Thus, the combined approach benefits from different sensitivities towards the vibrations caused by the isotopic labeling. In addition, these measurements also demonstrate the simultaneous execution of the IR and Raman measurements do not result in any signal interference (i.e., the measurements do not influence each other). Last but not least, this combined approach also offers the opportunity of in-situ validating data with an independent technique, should this be of interest.
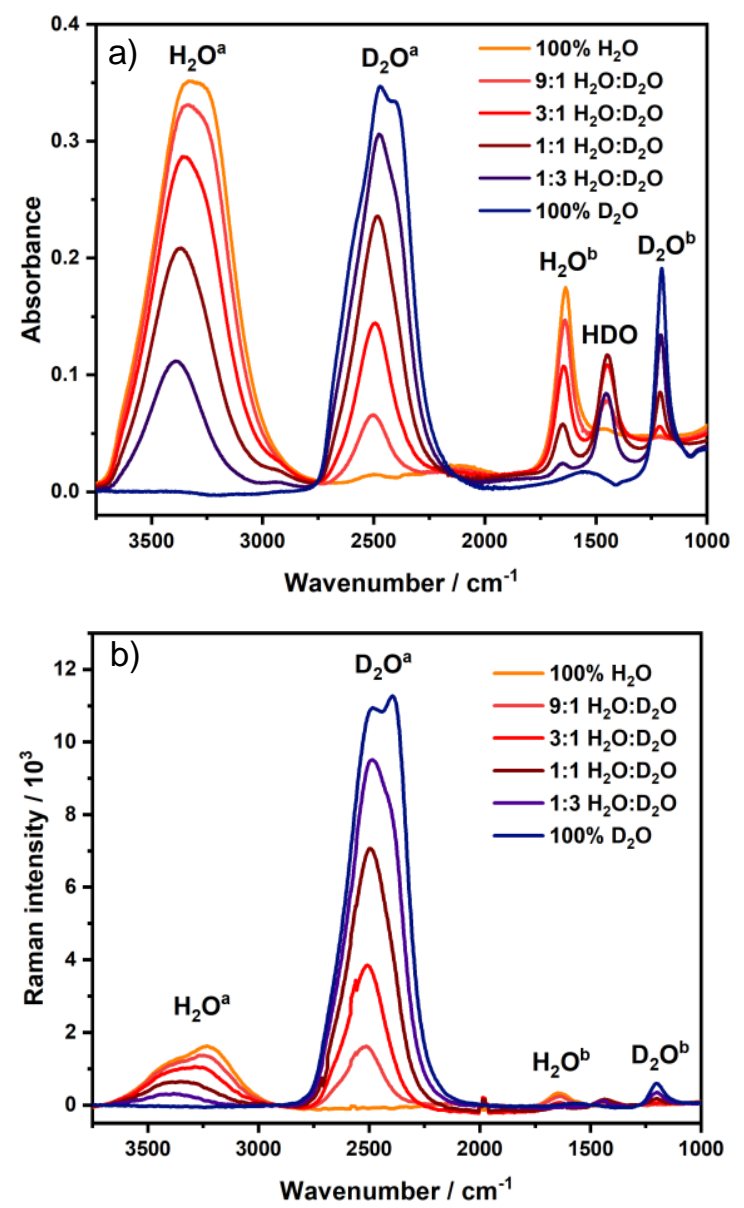

Figure 2. IR absorption spectra (a) and Raman spectra (b) of $\mathrm{D}_{2} \mathrm{O} / \mathrm{H}_{2} \mathrm{O}$ mixtures. The stretching vibrations are marked with ' $a$ ', the bending vibrations are labeled with ' $b$ '.

Bending vibrations $-\mathrm{H}_{2} \mathrm{O}^{\mathrm{b}}$ and $\mathrm{D}_{2} \mathrm{O}^{\mathrm{b}}$ at 1636 and $1208 \mathrm{~cm}^{-1}$ (IR), and at 1639 and $1204 \mathrm{~cm}^{-1}$ (Raman) - are more intense in the IR spectra vs. Raman measurements. Here, the different sensitivity towards particular vibrational modes for the two spectroscopic methods is once again evident. Thus, the combined measurements indeed act as orthogonal methods offering the opportunity to extend the range substances and vibrational modes that may be analyzed. 
Analysis of ammonium sulfate. In a second example, the capabilities of combined Raman and IR measurements for validation and orthogonality were demonstrated. Therefore, a variety of concentrations of ammonium sulfate in deionized water $(0,0.05,0.10,0.25,0.50$, 0.75 , and $1.00 \mathrm{M}$ ) were analyzed. Again, LOD and LOQ for each technique were derived.
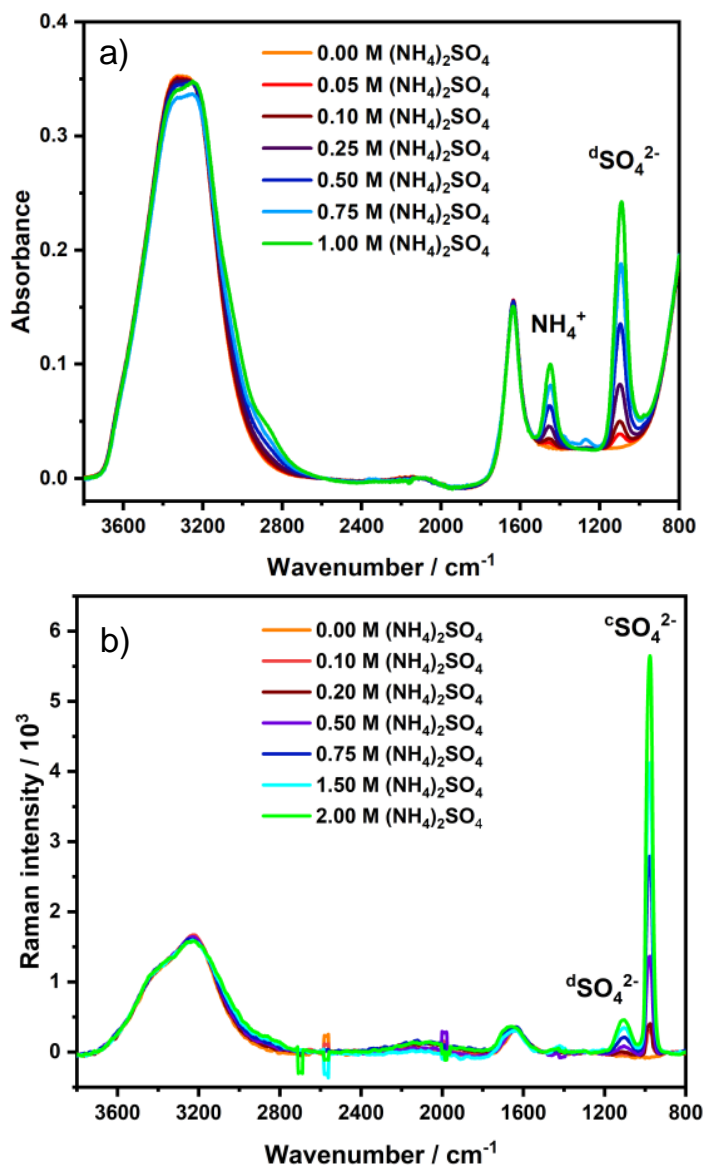

Figure 3. IR absorption spectra (a) and Raman spectra (b) of different concentrations of ammonium sulfate in deionized water. The vibrational modes of the sulfate ion are labeled with 'c' $\left(\nu_{1}\left(A_{1}\right)\right)$ and 'd' $\left(\nu_{3}\left(T_{2}\right)\right)$, respectively. ${ }^{38}$

Figure 3 shows exemplary spectra for both, IR (a) and Raman measurements (b) with the evaluated absorption bands ( ${ }^{\mathrm{S}} \mathrm{SO}_{4}{ }^{2+},{ }^{\mathrm{d}} \mathrm{SO}_{4}{ }^{2-}$ and $\mathrm{NH}_{4}{ }^{+}$). Obviously, the IR spectra show a strong water background across the entire spectra, while the Raman spectra are less influenced by water depicting clearly separated water bands at $3228 \mathrm{~cm}^{-1}$ and $1646 \mathrm{~cm}^{-1}$, respectively. Thus, Raman measurements provide less signal overlap in the spectral regions where, e.g., amide bands are occurring, which is beneficial/important for, e.g., biochemical applications studying proteins. ${ }^{48}$ Furthermore, Figure $3 \mathrm{~b}$ shows a second sulfate peak ${ }^{\mathrm{d}} \mathrm{SO}_{4}{ }^{2-}$ with less intensity near the analyzed sulfate band ' $\mathrm{SO}_{4}{ }^{2-}$. The 'free' and undistorted $\mathrm{SO}_{4}{ }^{2-}$ ion has a tetrahedron symmetry $\left(T_{\mathrm{d}}\right)$ associated with 9 active intramolecular vibrations that are all Raman active. In contrast, only the $T$ modes (i.e., $v_{3}\left(T_{2}\right)$ and $v_{4}\left(T_{2}\right)$ ) are IR active. ${ }^{38}$ Thus, for the performed calibration several vibrational modes of the sulfate ion were evaluated (see Figure 3). As there are vibrational modes that are active in both, IR and Raman or only in one of the two methods, respectively, and frequently exhibit differences in intensity, the developed multi-spectroscopic platform again benefits from increased access to different vibrational modes via IR and Raman, which may proof particularly important in complex matrices.

As shown in Figure 4, the calibration function of $\mathrm{SO}_{4}{ }^{2-}$ shows agreement between the IR and the Raman measurements. Both slopes $m$ of the linear regressions are almost equal, i.e., $m\left(\mathrm{SO}_{4}{ }^{2+}\right.$, Raman $)=1.01 \pm 0.01$ and $m\left(\mathrm{SO}_{4}{ }^{2+}, \mathrm{IR}\right)=1.00 \pm 0.01$, respectively indicating similar selectivity towards the sulfate ion although caused by different vibrational modes. The coefficient of determination is $R^{2}=0.9998$ for both linear regressions confirming excellent quality of the calibration functions in the examined concentration range. Once more, it is highlighted that the orthogonal application of two methods not only enables the interference-free identification and quantification of different molecular vibrations and thus species, but also allows validating the results with two independent methods. As both methods may be applied in-situ and at the same sample, also time may be saved for cross-checking data, which is an inherent benefit of the developed platform. Since both methods provide different spectral ranges where overlaps between the background matrix and the target analytes may occur, most spectral windows are available for evaluating target species without matrix interferences.

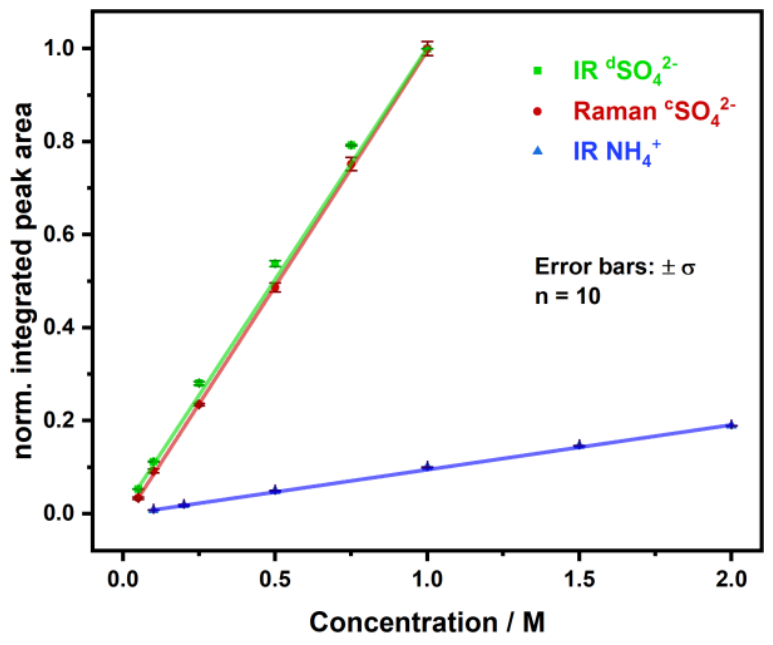

Figure 4. Calibration functions for sulfate and ammonium via simultaneous in-situ IR and Raman spectroscopy.

For IR, the LOD and LOQ was determined at $0.005 \mathrm{M}$ and $0.015 \mathrm{M}$, respectively for sulfate. In contrast, the Raman measurements provide six-fold higher values, i.e., $0.031 \mathrm{M}$ and $0.095 \mathrm{M}$. The LOD and LOQ was $0.104 \mathrm{M}$ and $0.316 \mathrm{M}$, respectively. The sensitivity for detecting ammonia and its quantification via the two spectroscopic methods was significantly lower, which is evident by the lower slope $m\left(\mathrm{NH}_{4}{ }^{+}, \mathrm{IR}\right)=0.096 \pm 0.001 \quad\left(R^{2}=0.9996\right)$. For the Raman measurements, no ammonium peak could be evaluated. Here the two methods provide complementary data and differences in sensitivity. If a particular species is not detectable by Raman spectroscopy in the observed concentration range, IR spectroscopy may be used and vice versa. This flexibility adds to the versatility 
of the developed analytical platform, especially in complex molecular scenarios.

Oxygen monitoring. Oxygen measurements were performed during each measurement. Figure 5 shows exemplarily the luminescence sensor response during a measurement period of $100 \mathrm{~s}$. The red line indicates the oxygen concentration in the gas phase, while the blue line shows the oxygen concentration monitored in the liquid phase. The straight line represents the calculated mean value of the respective measurement series during $100 \mathrm{~s}$. As the exemplary molecular systems analyzed herein do not cause any change of the oxygen concentration in both liquid and gas phase, the oxygen concentration should and indeed does - remain constant during the measured time, i.e., only slightly fluctuating around the mean value. This also indicates that the developed sampling cell is indeed gas-tight and providing excellent capabilities for future operation at inert conditions without influence of the ambient environment. It should be noted that monitoring of the gas phase is of particular importance as a complementary indicator for compositional changes in the liquid phase, e.g., during photocatalytic conversion studies. For example, the in-situ determination of the turn-over-number (TON) during water oxidation reactions ${ }^{16,49}$ or the monitoring of the oxygen concentration in water quality analysis ${ }^{15}$ rely on precise and continuous oxygen measurements, ideally without the need of expensive, off-line, and in part analyte- and time-consuming analytical techniques such gas chromatography (GC), electrochemical probes (e.g., Clark electrode), pressure transducers or volumetric methods, which in addition require diligent sampling and sample preparation..$^{50}$

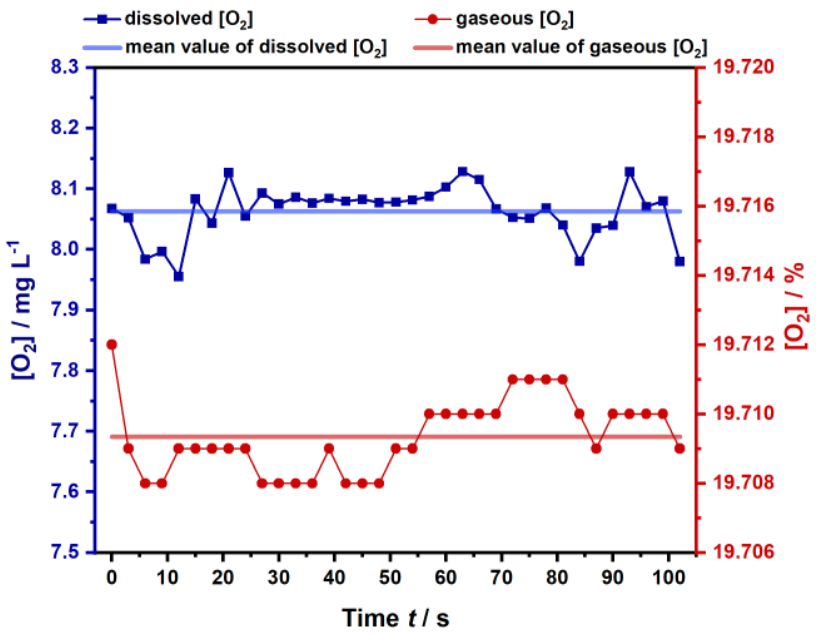

Figure 5. Luminescence sensor response of sensor 1 probing the headspace above the liquid sample (red), and sensor 2 immersed in the aqueous/liquid phase (blue).

2D Correlation Analysis. 2D correlation analysis especially benefits from a combined measurement assembly for the simultaneous collection of heterospectral data sets. This significantly simplifies the process of ensuring equal measurement conditions for the spectral moieties, which is an essential prerequisite for the application of 2DCOS. To this end, we applied heterospectral 2D correlation analysis to the data sets recorded in this study for demonstrating the possible application of this technique in future more challenging scenarios.

Figure 6 depicts the hetero-2DCOS obtained by correlating the Raman- and the IR data obtained in this study. As expected, the synchronous correlation spectrum is dominated by the intense sulfate band in the Raman spectrum at approx. $980 \mathrm{~cm}^{-1}$, which is correlated with the sulfate and ammonium signals at 1100 and $1450 \mathrm{~cm}^{-1}$, respectively. This indicates a synchronous, i.e. correlated change of these bands, which is different for, e.g., the water band at $1640 \mathrm{~cm}^{-1}$. While this is of course immediately evident for a simple system such as pure ammonium sulfate, in a more complex sample these highly correlated peaks would offer the opportunity to identify chemically related species within the spectra, even if they are highly convoluted.

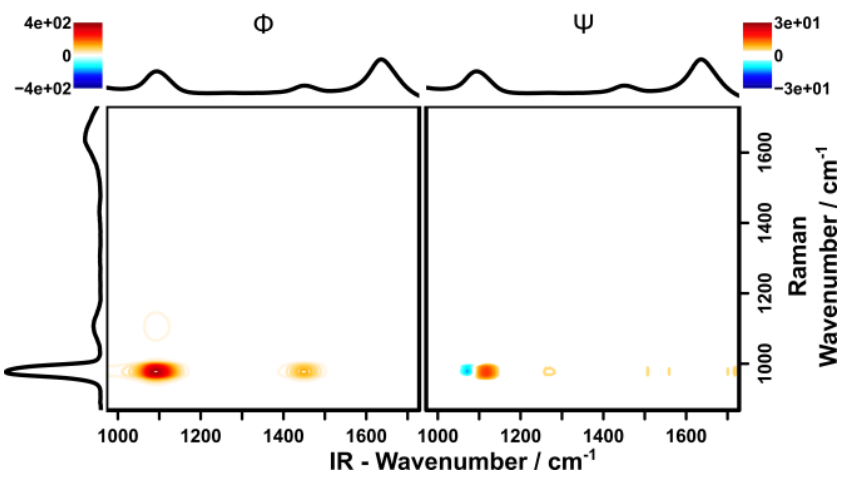

Figure 6. Synchronous (left) and asynchronous (right) hetero 2D correlation spectrum of ammonium sulfate solution between 0 and $2 \mathrm{M}$ in water. The $x$-axis shows the IR spectrum, the $y$-axis the Raman spectrum.

The asynchronous 2DCOS plot provides further insight into the multimodal measurement concept presented herein. Since 2DCOS is especially sensitive to minute differences in the perturbation dependency of signals, a simple one-component experiment as shown here may serve as an ideal benchmark to test for undesired, e.g., instrument-induced dependencies, since for a non-interacting one component system, no asynchronicity is expected. The asynchronous 2DCOS plot (Figure 6, right) shows one significantly asynchronous signal around (1100, 990) $\mathrm{cm}^{-1}$, and additional smaller signals along the $y=990 \mathrm{~cm}^{-1}$ parallel. Since these smaller signals do not have a corresponding signal in the synchronous spectrum, they can be attributed to noise residues and may thus be ignored from a molecular perspective. The signal at $(1100,990) \mathrm{cm}^{-1}$ can be explained by a shift of the asymmetric sulfate vibration signal to lower wavenumbers with increasing concentration, as reported in literature..$^{51}$ Such a band shift exerts itself in a characteristic positivenegative pattern in the asynchronous 2DCOS signal (a.k.a., 'butterfly' pattern). This is therefore a signal based on chemical signatures, and not induced, e.g., by the instrument. The absence of additional asynchronicity demonstrates that the developed multi-spectroscopic assembly provides an excellent tool for recording multimodal data sets without introducing differences in signal dependency between the two modalities 
The $\mathrm{H}_{2} \mathrm{O} / \mathrm{D}_{2} \mathrm{O}$ mixtures shows the potential of 2DCOS for discriminating uncorrelated and correlated changes between the complementary techniques, i.e., IR and Raman spectroscopy. Figure 7 depicts the synchronous and asynchronous hetero-2DCOS spectra of this data set. The synchronous 2DCOS is once again dominated by correlation patterns with the most intensive Raman band $\left(\mathrm{D}_{2} \mathrm{O}^{\mathrm{a}}\right)$ at $2484 \mathrm{~cm}^{-1}$ owing to its 9-fold higher intensities, as compared to the respective $\mathrm{H}_{2} \mathrm{O}^{\mathrm{a}}$ band. In addition, less intense correlation patterns can also be found along this line around $y=3235 \mathrm{~cm}^{-1}$. Positive correlation patterns of the $\mathrm{D}_{2} \mathrm{O}$ band with the corresponding $\mathrm{D}_{2} \mathrm{O}$-associated band in the IR spectrum $(2469,1209) \mathrm{cm}^{-1}$, as well as negative correlation patterns with the $\mathrm{H}_{2} \mathrm{O}$-associated bands at 3323 and $1653 \mathrm{~cm}^{-1}$ are evident. The inverse observation holds true for the correlation with the $\mathrm{H}_{2} \mathrm{O}^{\mathrm{a}}$ band, although here, only the patterns correlated with the stronger stretching vibrations are clearly evident. Importantly, the correlation pattern with the HDO bending vibration, which would be expected at $(1453,2484) \mathrm{cm}^{-1}$ is only weakly pronounced. This provides a first hint that the changes at these spectral positions, i.e., the increase in $\mathrm{D}_{2} \mathrm{O}$ signal and increase in HDO signal are not strongly correlated, and therefore, cannot be attributed to the same species.

Combining the synchronous with the asynchronous spectrum (Figure 7 , right) definitely confirms the assumptions derived from the synchronous spectrum. While for the correlation patterns of the bending vibrations (IR) of $\mathrm{H}_{2} \mathrm{O}$ and $\mathrm{D}_{2} \mathrm{O}$ with the $\mathrm{D}_{2} \mathrm{O}^{\mathrm{a}}$ stretching vibration (Raman) no asynchronicity occurs, a strong asynchronous cross-peak stretches around $(1453,2484) \mathrm{cm}^{-1}$, i.e., the $\mathrm{D}_{2} \mathrm{O} / \mathrm{HDO}$ correlation. This clearly evidences that the changes at these spectral positions cannot be associated with the same species. In an unknown system, such information is indispensable to ensure correct assignment of bands, and the associated changes in molecular species. In this multimodal setup, this could for example be used to associate IR signals that are highly convoluted with a strong and well-known Raman band of a target species, therefore enabling the tracking of changes to this species even in a convoluted sample matrix.

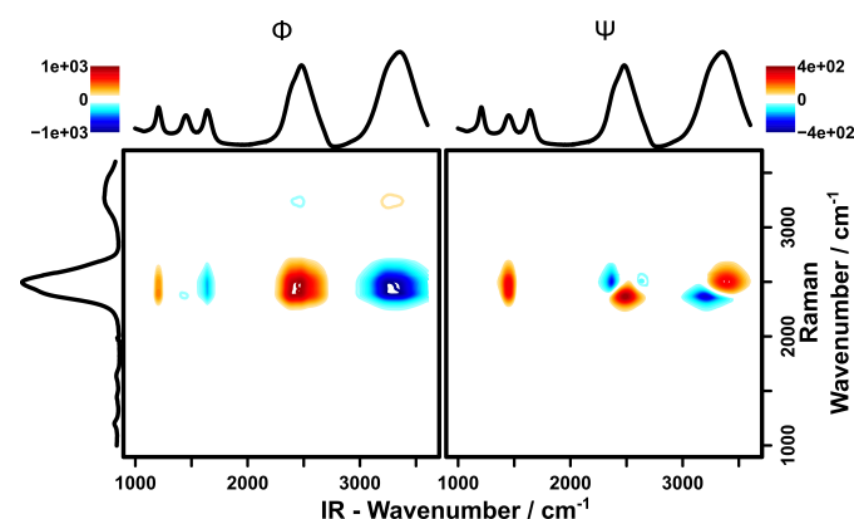

Figure 7. Synchronous (left) and asynchronous (right) hetero $2 \mathrm{D}$ correlation spectrum of mixtures between $\mathrm{H}_{2} \mathrm{O}$ and $\mathrm{D}_{2} \mathrm{O}$. The $x$-axis shows the IR spectrum, the $y$-axis the Raman spectrum.
Furthermore, the asynchronous spectrum once again reveals two 'butterfly' patterns associated with the $\mathrm{D}_{2} \mathrm{O}^{\mathrm{a}}$ stretching vibration, and with the stretching vibrations of $\mathrm{H}_{2} \mathrm{O}$ and $\mathrm{D}_{2} \mathrm{O}$ in the IR spectrum. These patterns once again result from band shifts of the stretching vibrations due to the change in hydrogen bonding environment depending on the $\mathrm{D}_{2} \mathrm{O} / \mathrm{H}_{2} \mathrm{O}$ ratio. These effects are known from literature ${ }^{37}$, and are therefore once again indicating molecular information rather than instrument-induced artefacts.

\section{CONCLUSIONS AND OUTLOOK}

The present study demonstrates the successful integration of three optical measurement techniques into a single measurement assembly enabling simultaneous in-situ IRATR, Raman, and luminescence quenching measurements in a sample volume of $7 \mathrm{~mL}$ providing complementary and/or validating information, respectively.

The sample cell was designed such that a modular adaption/addition of a variety of optical techniques to a basic assembly of a single-bounce diamond IR-ATR device coupled to an FTIR spectrometer is facilitated. The modularity of the concept enables that essentially any fiberoptically coupled Raman spectrometer may be used, as the cell lid and the probe adapters may be readily modified according to the dimensions of the utilized probe. The platform is also constructed in a way that other fiberoptically coupled tool such as light sources (e.g., LEDs with different emission wavelengths, etc.) could be readily inserted. This approach may be used for triggering, e.g., light-driven reactions, as required for studying photocatalysis in molecular detail. The gas-tightness of the assembly provides the opportunity for facilitating inert reaction conditions (e.g., working under argon, etc.), as frequently required during catalytic reactions, but also for precisely determining the evolved oxygen concentration when determining TONs or when monitoring changes in oxygen levels as an indicator of compositional changes in solution.

Furthermore, the achieved analytical figures-of-merit evidence that the obtained measurements - because of the robustness of the developed multi-spectroscopic assembly - are highly reproducible, as confirmed by the quality of the obtained calibration functions and by the absence of instrument-attributed asynchronicity in the 2D correlation analysis results. Two exemplary molecular systems $-\mathrm{H}_{2} \mathrm{O} / \mathrm{D}_{2} \mathrm{O}$ and dissolved ammonium sulfate were selected for verifying the utility and functionality of the system. In addition, the developed approach allows for in-situ validations via complementary yet independent analytical techniques (i.e., IR vs. Raman) without timeconsuming additional experiments. The orthogonality of the achieved results is clearly evident for both exemplary systems, as there are different Raman and/or IR active bands also varying in intensity, respectively. Thus, different spectral overlaps of matrix and target analyte bands are provided, which expands the interference-free spectral range that may be used for analyzing complex molecular mixtures via the developed multi-spectroscopic platform. 
During future studies, the developed platform will be used for on-line monitoring of environmental pollution scenarios, and for in-situ following molecular changes during light-driven photocatalytic reactions. The modular system concept also allows operating the sample cell in a flowthrough mode enabling dynamic studies at controlled flow conditions.

Furthermore, it is anticipated that a similar device will be adapted for replacing conventional FTIR techniques with quantum/interband cascade laser-based spectroscopies combined with thin-film waveguide technology promising further enhanced sensitivity in even smaller sample volumes, as fundamentally shown during previous studies of our research team. ${ }^{1,6,37}$

Last but not least, the simultaneous execution of orthogonal spectroscopies at the same sample clearly simplifies data fusion approaches, as well as using (heterogeneous) 2D correlation methods (i.e., IR and Raman data) for extracting more information from complex data sets, as already demonstrated for the model systems herein. The simultaneous execution of the measurements ensures that the same experimental and/or reaction conditions in terms of temperature, light power, ambient conditions, etc. are present, which is a prerequisite for useful 2D correlation analysis, and can be applied for more realistic and complex systems in future.

\section{AUTHOR INFORMATION}

\section{Corresponding Author}

* Boris Mizaikoff - Institute of Analytical and Bioanalytical Chemistry, Ulm University, Albert-Einstein-Allee 11, 89081 Ulm, Germany; http://orcid.org/0000-0002-5583-7962; Email: boris.mizaikoff@uni-ulm.de

\section{Authors}

Sarah Klingler - Institute of Analytical and Bioanalytical Chemistry, Ulm University, Albert-Einstein-Allee 11, 89081 Ulm, Germany; http://orcid.org/0000-0002-7919-093X; Email: sarah.klingler@uni-ulm.de

Julian Hniopek - Department of Spectroscopy/lmaging, Leibniz-Institute of Photonic Technologies, Jena, Germany; Institute of Physical Chemistry \& Abbe Center of Photonics, Friedrich Schiller University Jena, Jena, Germany, Email: julian.hniopek@uni-jena.de

Robert Stach -Hahn-Schickard, Sedanstraße 14, 89077 Ulm, Germany; Email: robert.stach@hahn-schickard.de Michael Schmitt - Department of Spectroscopy/lmaging, Leibniz-Institute of Photonic Technologies, Jena, Germany; Institute of Physical Chemistry \& Abbe Center of Photonics, Friedrich Schiller University Jena, Jena, Germany, Email: m.schmitt@uni-iena.de

Jürgen Popp - Department of Spectroscopy/lmaging, Leibniz-Institute of Photonic Technologies, Jena, Germany; Institute of Physical Chemistry \& Abbe Center of Photonics, Friedrich Schiller University Jena, Jena, Germany, Email: juergen.popp@uni-jena.de

\section{Present Addresses}

a Institute of Analytical and Bioanalytical Chemistry, Ulm University, Albert-Einstein-Allee 11, 89081 Ulm, Germany
${ }^{b}$ Department of Spectroscopy/Imaging, Leibniz-Institute of Photonic Technologies, Jena, Germany

${ }^{c}$ Institute of Physical Chemistry \& Abbe Center of Photonics, Friedrich Schiller University Jena, Jena, Germany

d Hahn-Schickard, Sedanstraße 14, 89077 Ulm, Germany

\section{Author Contributions}

S.K. designed and performed the experiments. S.K. evaluated the data and wrote the article. B.M designed and supervised the experiments. J.H. and S.K. performed the $2 \mathrm{D}$ correlation analysis and interpreted the 2D data. R.S., M.S., J.P. and B.M. proofread the article. All authors have given approval to the final version of the manuscript.

\section{Funding Sources}

The funding by the Deutsche Forschungsgemeinschaft DFG (TRR234 "CataLight", project ID 364549901 (project C2) is greatly acknowledged.

\section{Notes}

The authors declare no competing financial interest.

\section{ACKNOWLEDGMENT}

The authors acknowledge the support of the members of the Institute of Analytical and Bioanalytical Chemistry (IABC) at Ulm University. This study is funded by the Deutsche Forschungsgemeinschaft DFG (TRR234 "CataLight", project ID 364549901 (project C2). The Machine Shop at Ulm University is thanked for support during prototype development.

\section{REFERENCE}

(1) Sieger, M.; Haas, J.; Jetter, M.; Michler, P.; Godejohann M.; Mizaikoff, B. Mid-Infrared Spectroscopy Platform Based on GaAs/AlGaAs Thin-Film Waveguides and Quantum Cascade Lasers. Anal. Chem. 2016, 88 (5), 2558-2562. https://doi.org/10.1021/acs.analchem.5b04144.

Haas, J.; Mizaikoff, B. Advances in Mid-Infrared Spectroscopy for Chemical Analysis. Annu. Rev. Anal. Chem. 2016, 9, 45-68. https://doi.org/10.1146/annurevanchem-071015-041507.

(3) Mizaikoff, B. Waveguide-Enhanced Mid-Infrared Chem/Bio Sensors. Chem. Soc. Rev. 2013, 42 (22), 8683-8699. https://doi.org/10.1039/c3cs60173k.

(4) Haas, J.; Müller, A.; Sykora, L.; Mizaikoff, B. Analytica Performance of $\mu$-Groove Silicon Attenuated Total Reflection Waveguides. Analyst 2019, 144 (10), 33983404. https://doi.org/10.1039/c9an00417c.

(5) Stach, R.; Krebs, P.; Jones, F.; Mizaikoff, B. Observing Non-Classical Crystallisation Processes in Gypsum via Infrared Attenuated Total Reflectance Spectroscopy. CrystEngComm 2017, 19 (1), 14-17. https://doi.org/10.1039/c6ce01787h.

(6) Janotta, M.; Vogt, F.; Voraberger, H. S.; Waldhauser, W. Lackner, J. M.; Stotter, C.; Beutl, M.; Mizaikoff, B. Direct Analysis of Oxidizing Agents in Aqueous Solution with Attenuated Total Reflectance Mid-Infrared Spectroscopy and Diamond-like Carbon Protected Waveguides. Anal. Chem. 2004, 76 (2), 384-391. https://doi.org/10.1021/ac034699d.

(7) Andanson, J. M.; Baiker, A. Exploring Catalytic Solid/Liquid Interfaces by in Situ Attenuated Total Reflection Infrared Spectroscopy. Chem. Soc. Rev. 2010, 39 (12), 4571-4584. https://doi.org/10.1039/b919544k.

(8) Kim, S. S.; Young, C.; Mizaikoff, B. Miniaturized MidInfrared Sensor Technologies. Anal. Bioanal. Chem. 2008 390 (1), 231-237. https://doi.org/10.1007/s00216-0071673-5.

(9) Haas, J.; Catalán, E. V.; Piron, P.; Karlsson, M.; Mizaikoff, B. Infrared Spectroscopy Based on Broadly Tunable Quantum Cascade Lasers and Polycrystalline Diamond 
Waveguides. Analyst 2018, 143 (21), 5112-5119. https://doi.org/10.1039/c8an00919h.

(10) Wartewig, S.; Neubert, R. H. H. Pharmaceutical Applications of Mid-IR and Raman Spectroscopy. Adv. Drug Deliv. Rev. 2005, 57 (8), 1144-1170. https://doi.org/10.1016/j.addr.2005.01.022.

(11) Rostron, P.; Gaber, S.; Gaber, D. Raman Spectroscopy , Review. Int. J. Eng. Tech. Res. 2016, 0869 (1), 50-64.

(12) Ember, K. J. I.; Hoeve, M. A.; McAughtrie, S. L.; Bergholt, M. S.; Dwyer, B. J.; Stevens, M. M.; Faulds, K.; Forbes, S. J.; Campbell, C. J. Raman Spectroscopy and Regenerative Medicine: A Review. npj Regen. Med. 2017, 2 (1). https://doi.org/10.1038/s41536-017-0014-3.

(13) Smith, G. D.; Clark, R. J. H. Raman Microscopy in Archaeological Science. J. Archaeol. Sci. 2004, 31 (8), 1137-1160. https://doi.org/10.1016/j.jas.2004.02.008.

(14) Najah, A.; El-Shafie, A.; Karim, O. A.; El-Shafie, A. H. Performance of ANFIS versus MLP-NN Dissolved Oxygen Prediction Models in Water Quality Monitoring. Environ. Sci. Pollut. Res. 2014, 21 (3), 1658-1670. https://doi.org/10.1007/s11356-013-2048-4.

(15) Kannel, P. R.; Lee, S.; Lee, Y. S.; Kanel, S. R.; Khan, S. P. Application of Water Quality Indices and Dissolved Oxygen as Indicators for River Water Classification and Urban Impact Assessment. Environ. Monit. Assess. 2007, 132 (13), 93-110. https://doi.org/10.1007/s10661-006-9505-1.

(16) Huber, F. L.; Amthor, S.; Schwarz, B.; Mizaikoff, B.; Streb, C.; Rau, S. Multi-Phase Real-Time Monitoring of Oxygen Evolution Enables: In Operando Water Oxidation Catalysis Studies. Sustain. Energy Fuels 2018, 2 (9), 1974-1978. https://doi.org/10.1039/c8se00328a.

(17) McDonagh, C.; Kolle, C.; McEvoy, A. K.; Dowling, D. L.; Cafolla, A. A.; Cullen, S. J.; MacCraith, B. D. Phase Fluorometric Dissolved Oxygen Sensor. Sensors Actuators, B Chem. 2001, 74 (1-3), 124-130. https://doi.org/10.1016/S0925-4005(00)00721-8.

(18) MacCraith, B. D.; McDonagh, C. M.; O'Keeffe, G.; Keyes, E. T.; Vos, J. G.; O'Kelly, B.; McGilp, J. F. Fibre Optic Oxygen Sensor Based on Fluorescence Quenching of Evanescent-Wave Excited Ruthenium Complexes in SolGel Derived Porous Coatings. Analyst 1993, 4 (118), 385388. https://doi.org/10.1039/AN9931800385.

(19) Chu, C. S.; Lo, Y. L.; Sung, T. W. Review on Recent Developments of Fluorescent Oxygen and Carbon Dioxide Optical Fiber Sensors. Photonic Sensors 2011, 1 (3), 234250. https://doi.org/10.1007/s13320-011-0025-4.

(20) Jorge, P. A. S.; Caldas, P.; Rosa, C. C.; Oliva, A. G.; Santos, J. L. Optical Fiber Probes for Fluorescence Based Oxygen Sensing. Sensors Actuators, B Chem. 2004, 103 (1-2), 290-299. https://doi.org/10.1016/j.snb.2004.04.086.

(21) Wang, X. D.; Wolfbeis, O. S. Fiber-Optic Chemical Sensors and Biosensors (2013-2015). Anal. Chem. 2016, 88 (1), 203-227. https://doi.org/10.1021/acs.analchem.5b04298.

(22) Chodavarapu, V. P.; Shubin, D. O.; Bukowski, R. M.; Titus, A. H.; Cartwright, A. N.; Bright, F. V. CMOS-Based Phase Fluorometric Oxygen Sensor System. IEEE Trans. Circuits Syst. I Regul. Pap. 2007, 54 (1), 111-118. https://doi.org/10.1109/TCSI.2006.888680.

(23) Grabow, K.; Bentrup, U. Homogeneous Catalytic Processes Monitored by Combined in Situ ATR-IR, UV-VIS, and Raman Spectroscopy. ACS Catal. 2014, 4 (7), 21532164. https://doi.org/10.1021/cs500363n.

(24) Zhao, Y.; Yuan, J.; Ji, Z.; Wang, J.; Rohani, S. Combined Application of in Situ FBRM, ATR-FTIR, and Raman on Polymorphism Transformation Monitoring during the Cooling Crystallization. Ind. Eng. Chem. Res. 2012, 51 (38), 12530-12536. https://doi.org/10.1021/ie301241h.

(25) Hu, Y.; Liang, J. K.; Myerson, A. S.; Taylor, L. S. Crystallization Monitoring by Raman Spectroscopy: Simultaneous Measurement of Desupersaturation Profile and Polymorphic Form in Flufenamic Acid Systems. Ind. Eng. Chem. Res. 2005, 44 (5), 1233-1240. https://doi.org/10.1021/ie049745u.

(26) Ono, T.; Ter Horst, J. H.; Jansens, P. J. Quantitative Measurement of the Polymorphic Transformation of L-
Glutamic Acid Using in-Situ Raman Spectroscopy. Cryst. Growth Des. 2004, 4 (3), 465-469. https://doi.org/10.1021/cg0342516.

Liu, W.; Wei, H.; Black, S. An Investigation of the Transformation of Carbamazepine from Anhydrate to Hydrate Using in Situ FBRM and PVM. Org. Process Res. Dev. 2009, 13 (3), 494-500. https://doi.org/10.1021/op8002773.

(28) Cornel, J.; Mazzotti, M. Calibration-Free Quantitative Application of in Situ Raman Spectroscopy to a Crystallization Process. Anal. Chem. 2008, 80 (23), 92409249. https://doi.org/10.1021/ac801606z.

(29) Bentrup, U. Combining in Situ Characterization Methods in One Set-up: Looking with More Eyes into the Intricate Chemistry of the Synthesis and Working of Heterogeneous Catalysts. Chem. Soc. Rev. 2010, 39 (12), 4718-4730. https://doi.org/10.1039/b919711g.

(30) Tinnemans, S. J.; Mesu, J. G.; Kervinen, K.; Visser, T.; Nijhuis, T. A.; Beale, A. M.; Keller, D. E.; Van Der Eerden, A. M. J.; Weckhuysen, B. M. Combining Operando Techniques in One Spectroscopic-Reaction Cell: New Opportunities for Elucidating the Active Site and Related Reaction Mechanism in Catalysis. Catal. Today 2006, 113 (1-2), 3-15. https://doi.org/10.1016/j.cattod.2005.11.076.

(31) O'Sullivan, B.; Glennon, B. Application of in Situ FBRM and ATR-FTIR to the Monitoring of the Polymorphic Transformation of D-Mannitol. Org. Process Res. Dev. 2005, 9 (6), 884-889. https://doi.org/10.1021/op0500887.

(32) Zhao, Y.; Bao, Y.; Wang, J.; Rohani, S. In Situ Focused Beam Reflectance Measurement (FBRM), Attenuated Total Reflectance Fourier Transform Infrared (ATR-FTIR) and Raman Characterization of the Polymorphic Transformation of Carbamazepine. Pharmaceutics 2012, 4 (1), https://doi.org/10.3390/pharmaceutics4010164.

(33) Schöll, J.; Bonalumi, D.; Vicum, L.; Mazzotti, M.; Müller, M. In Situ Monitoring and Modeling of the Solvent-Mediated Polymorphic Transformation of L-Glutamic Acid. Cryst. Growth Des. 2006, 6 (4), 881-891. https://doi.org/10.1021/cg0503402.

(34) Garland, M.; Li, C. A Review of BTEM Analysis for Catalytic Studies and a Recent Homogeneous Catalytic Example. Top. Catal. 2009, 52 (10), 1334-1341. https://doi.org/10.1007/s11244-009-9311-4.

(35) Noda, I. Generalized Two-Dimensional Correlation Method Applicable to Infrared, Raman, and Other Types of Spectroscopy. Appl. Spectrosc. 1993, 47 (9), 1329-1336.

(36) Geitner, R.; Götz, S.; Stach, R.; Siegmann, M.; Krebs, P.; Zechel, S.; Schreyer, K.; Winter, A.; Hager, M. D.; Schubert, U. S.; Gräfe, S.; Dietzek, B.; Mizaikoff, B.; Schmitt, M.; Popp, J. Hydrogel-Embedded Model Photocatalytic System Investigated by Raman and IR Spectroscopy Assisted by Density Functional Theory Calculations and TwoDimensional Correlation Analysis. J. Phys. Chem. A 2018, $122 \quad$ (10), 2677-2687. https://doi.org/10.1021/acs.jpca.7b12648.

(37) Neubauer, D.; Korbmacher, J.; Frick, M.; Kiss, J.; Timmler, M.; Dietl, P.; Wittekindt, O. H.; Mizaikoff, B. Deuterium Oxide Dilution: A Novel Method to Study Apical Water Layers and Transepithelial Water Transport. Anal. Chem. 2013, $\quad 85 \quad$ (9), $4247-4250$. https://doi.org/10.1021/ac4002723.

(38) Pye, C. C.; Rudolph, W. W. An Ab Initio and Raman Investigation of Sulfate Ion Hydration. J. Phys. Chem. A 2001, 105 (5), 905-912. https://doi.org/10.1021/jp003253n. Chien, S. H.; Teixeira, L. A.; Cantarella, H.; Rehm, G. W.; Grant, C. A.; Gearhart, M. M. Agronomic Effectiveness of Granular Nitrogen/Phosphorus Fertilizers Containing Elemental Sulfur with and without Ammonium Sulfate: A Review. Agron. J. 2016, 108 (3), 1203-1213. https://doi.org/10.2134/agronj2015.0276.

(40) Hniopek, J.; Schmitt, M.; Popp, J.; Bocklitz, T. W. PC2DCOS: A Principal Component Base Approach to TwoDimensional Correlation Spectroscopy. Appl. Spectrosc. 2020, 74 (4), 460-472. 
https://doi.org/10.1177/0003702819891194.

(41) Geitner, R.; Fritzsch, R.; Popp, J.; Bocklitz, T. W. Corr2d: Implementation of Two-Dimensional Correlation Analysis in R. J. Stat. Softw. 2019, 90 (August 2019). https://doi.org/10.18637/jss.v090.i03.

(42) Venyaminov, S. Y.; Prendergast, F. G. Water ( $\mathrm{H} 2 \mathrm{O}$ and D2O) Molar Absorptivity in the 1000-4000 Cm-1 Range and Quantitative Infrared Spectroscopy of Aqueous Solutions. Anal. Biochem. 1997, 248 (2), 234-245. https://doi.org/10.1006/abio.1997.2136.

(43) Brooker, M. H.; Hancock, G.; Rice, B. C.; Shapter, J. Raman Frequency and Intensity Studies of Liquid $\mathrm{H} 2 \mathrm{O}$, H218O and D2O. J. Raman Spectrosc. 1989, 20 (10), 683694. https://doi.org/10.1002/jrs.1250201009.

(44) Venkateswarlu, P.; Bist, H. D.; Jain, Y. S. Laser Excited Raman Spectrum of Ammonium Sulfate Single Crystal. J. Raman Spectrosc. 1975, 3 (2-3), 143-151. https://doi.org/10.1002/jrs.1250030205.

(45) Cziczo, D. J.; Abbatt, J. P. D. Deliquescence, Efflorescence, and Supercooling of Ammonium Sulfate Aerosols at Low Temperature: Implications for Cirrus Cloud Formation and Aerosol Phase in the Atmosphere. J. Geophys. Res. Atmos. 1999, 104 (D11), 13781-13790. https://doi.org/10.1029/1999JD900112.

(46) Fontana, M. D.; Mabrouk, K. Ben; Kauffmann, T. H. Raman Spectroscopic Sensors for Inorganic Salts. Spectrosc. Prop. Inorg. Organomet. Compd. 2013, 44 (September
2018), 40-67. https://doi.org/10.1039/9781849737791 00040.

(47) Colles, M. J.; Walrafen, G. E.; Wecht, K. W. Stimulated Raman Spactra from $\mathrm{H} 2 \mathrm{O}, \mathrm{D} 2 \mathrm{O}, \mathrm{HDO}$ and Solutions of $\mathrm{NaClO} 4$ in $\mathrm{H} 2 \mathrm{O}$ and D2O. Chem. Phys. Lett. 1970, 4 (10), 621-624.

(48) Bandekar, J. Amide Modes and Protein Conformation. Biochim. Biophys. Acta (BBA)/Protein Struct. Mol. 1992 1120 (2), 123-143. https://doi.org/10.1016/01674838(92)90261-B.

(49) Li, B.; Li, F.; Bai, S.; Wang, Z.; Sun, L.; Yang, Q.; Li, C. Oxygen Evolution from Water Oxidation on Molecular Catalysts Confined in the Nanocages of Mesoporous Silicas. Energy Environ. Sci. 2012, 5 (8), 8229-8233. https://doi.org/10.1039/c2ee22059h.

(50) Wolfbeis, O. S. Luminescent Sensing and Imaging of Oxygen: Fierce Competition to the Clark Electrode. BioEssays 2015, 37 (8), 921-928. https://doi.org/10.1002/bies.201500002.

(51) Rudolph, W. W. Hydration and Water - Ligand Replacement in Aqueous Cadmium ( II ) Sulfate Solution A Raman and Infrared Study Hydration and Water - Ligand Replacement in Aqueous Cadmium ( II ) Sulfate Solution A Raman and Infrared Study. 2014, 3 (February 1998). https://doi.org/10.1039/a705212j. 